\title{
Elastic Cross-Section of Positron Scattering from Helium Ions
}

\author{
Mahasen. M. Abdel-Mageed \\ (Physics Department, Faculty of Science, Ain Shams University, Abbassia, Cairo11566, Egypt)
}

\begin{abstract}
The collisions of positrons with singly charged helium ion $\mathrm{He}^{+}(1 \mathrm{~s})$ at ground state have been investigated using Harris-Nesbet variational method. This method is demonstrated for total elastic cross sections which corresponding to $S$-, $P$-, and $D$-wave scatterings below positronium formation threshold. The incident energies is considered up to $40 \mathrm{eV}$ (positron momentum $k \cong 1.7$ a.u.). The results show oscillating behavior of the cross sections in $S$-wave, where the existence of a resonance is expected, the first peak starts in $S$-wave and moving toward higher values of $k$ as the angular momentum $L$ increases. We encourage the experimental physicists to carry out further positron-ions experiments.
\end{abstract}

Keywords: Elastic Collisions, Positrons, Helium Ion, L-wave, Cross sections.

\section{INTRODUCTION}

The scattering of positrons by atomic ions has gained significant importance because the positron has being a positively-charged probe, offers a more sensitive test of our ability to understand atomic interactions than the electron does [1]. Low-energy positron scattering on positive ions are quite different from those of positrons on neutral atoms. For neutral species the long-range positron interaction is attractive, while for positive ions the dominant long range potential is Coulomb repulsive. In addition the typical energy scale that characterizes the positron interaction with positive ions is significantly larger than that of positron-atom systems, [e.g.; the positronium Ps formation energy threshold with of hydrogen is $6.8 \mathrm{eV}$ while it is $47.6 \mathrm{eV}$ for $\mathrm{He}^{+}$] [2]. Harris-Nesbet method is used to determine with great success the doubly-excited-state resonances of He below the $\mathrm{N}=2$ ionization threshold [3] .The Kohn variational method is used to study positron scattering in $\mathrm{He}^{+}[4-5]$. A coupled channel approximation has been employed to calculate cross sections for positron collisions with some hydrogen like atoms below positronium formation [6-7]. In this paper we use the HarrisNesbet method to calculate the scattering cross section of positron with singly ionized helium.

\section{THEORY}

The total Hamiltonian $\mathrm{H}$ for the $\mathrm{e}^{+}-\mathrm{He}^{+}$system, with the energy expressed in Rydberg is given by:

$$
\begin{gathered}
H=T+V \\
T=-\nabla_{1}-\nabla_{2} \\
V=-\frac{2 Z}{r_{1}}+\frac{Z}{r_{2}}-\frac{2}{r_{12}}
\end{gathered}
$$

where indices 1 and 2 refer to the coordinates of the electron and the positron, respectively, $\mathrm{Z}$ is the nucleus charge of the helium, $\boldsymbol{r}_{1}, \boldsymbol{r}_{2}$ are the position vectors for electron and positron, respectively, $\boldsymbol{r}_{12}=\left|\boldsymbol{r}_{1}-\boldsymbol{r}_{2}\right|$, and $\boldsymbol{V}$ is the static Coulomb potential

1. Harris-Nesbet Variational Method

Harris proposed a new expansion method by taking the advantage of the singularities in the Kohn method and then proposed a new variational method which is suitable for applications at continuous energies to avoid the singularities appearing in the Kohn variational method, Harris proposed a new expansion method by taking the advantage of the singularities in the Kohn method and then proposed a new variational method which is suitable for applications at continuous energies. For scattering system with a Hamiltonian $\mathrm{H}$ at energy $\mathrm{E}$, the Schrodinger equation is

$$
(H-E) \Psi=0
$$

where $\Psi$ is a total scattering wavefunction, which can be written as [8]

$$
\Psi=\alpha_{0}\left(\phi_{s}+S\right)+\alpha_{1}\left(\phi_{c}+C\right)
$$

Thus the functional of the collision process should be, as usual, defined as [9]

$$
\langle\Psi|H-E| \Psi\rangle=V R
$$


where S and C are quadratically integrable, $\phi$ is a short range function which can expanded in terms of $\phi_{\mathrm{k}}$

$$
\phi_{s}=\sum_{k} B_{k} \phi_{k} \quad \phi_{c}=\sum_{k} B_{k} \phi_{k}
$$

These function satisfy the following variations

$$
\begin{aligned}
& \alpha_{1}<\phi_{k}|H-E| \phi_{c}+C>=0 \\
& \alpha_{o}<\phi_{k}|H-E| \phi_{s}+S>=0
\end{aligned}
$$

Where

$$
\begin{aligned}
& A_{k}=\left(E-E_{k}\right)^{-1}<\phi_{k}|H-E| S> \\
& B_{k}=\left(E-E_{k}\right)^{-1}<\phi_{k}|H-E| C>
\end{aligned}
$$

Eqs. (7) and (8) above enable the reduction of the functional in eq.(6) as:

Where

$$
V R=m_{o o} \alpha_{o}^{2}+\left(m_{o 1}+m_{1 o}\right) \alpha_{o} \alpha_{1}+m_{11} \alpha_{1}^{2}
$$

$$
\begin{gathered}
\boldsymbol{m}_{\mathrm{oO}}=<S|H-E| \phi_{s}+S>\quad, \quad m_{01}=<S|H-E| \phi_{c}+C>, \\
\boldsymbol{m}_{10}=<C|H-E| \phi_{s}+C>\quad \text { and } \quad m_{11}=<C|H-E| \phi_{c}+C>
\end{gathered}
$$

The bound wave function considered as a Slater wave function, while, the hydrogen-like-ion targets, the $S$ function for the scattering channel can considered as [10]:

$$
S\left(\boldsymbol{r}_{2}\right)=k \boldsymbol{J}_{l}\left(k \boldsymbol{r}_{2}\right)
$$

$\boldsymbol{J}_{\boldsymbol{l}}\left(k r_{2}\right)$ is the so-called Coulomb-Bessel function. The Armstead form for the $\boldsymbol{C}$ function is chosen [10-11]

$$
C\left(\boldsymbol{r}_{2}\right)=k\left[\frac{\eta}{l+1}\right] J_{l}\left(k \boldsymbol{r}_{2}\right)+\sqrt{1+\left(\frac{\eta}{l+1}\right)^{2}+} J_{l+1}\left(k \boldsymbol{r}_{2}\right)
$$

The inverse of the reactance matrix can be written as

$$
\boldsymbol{R}_{k}^{-1}=2\left(\boldsymbol{m}_{o o}-\boldsymbol{m}_{01}^{+} \boldsymbol{m}_{11}^{-1} \boldsymbol{m}_{o 1}\right)
$$

The transition matrix, T, is related to the reactance matrix $R_{k}$ by [8]

$$
T_{k}=R_{k}\left(I-\tilde{i} R_{k}\right)^{-1},
$$

where $I$ is unit matrix and $\tilde{i}=\sqrt{-1}$.

The partial cross-sections corresponding to the angular momentum $\ell$, i.e. $\sigma_{l}{ }^{\prime} s$ are determined (in $\pi a_{0}^{2}$ units) by:

$$
\sigma_{l}=\frac{4(2 \ell+1)}{k^{2}}|T|^{2}
$$

The total cross-sections are calculated by:

$$
\sigma=\sum_{\ell=0}^{\infty} \sigma_{l}
$$

\section{RESULTS AND DISCUSSION}

The mathematical formalism of the preceding section has been implemented to the collisions of positrons with singly ionized helium. The parameters in Eq. (10) are obtained using optimization technique. The reactance matrix and transition matrix were determined to calculate the partial and total cross section. 
Table 1. The Variation of the partial elastic cross sections (in $\pi a_{0}^{2}$ ) of $\mathrm{e}^{+}-\mathrm{H}^{+}$

scattering, with positron momentum in (a.u. ), for S-, P- and D- waves.

\begin{tabular}{l|lll}
\hline $\mathrm{k}\left(\mathrm{a}_{\mathrm{o}}{ }^{-1}\right)$ & S-wave $(\mathrm{l}=0)$ & $\mathrm{P}-w a v e(1=1)$ & D-wave $(\mathrm{l}=2)$ \\
\hline 0.25 & $2.1185(-12)$ & $6.0887(-12)$ & $5.73735(-12)$ \\
0.35 & $5.4831(-11)$ & $1.03277(-11)$ & $8.61685(-11)$ \\
0.45 & $8.15173(-10)$ & $3.51255(-10)$ & $4.39825(-10)$ \\
0.70 & $5.33433(-5)$ & $4.52981(-5)$ & $1.6099 \mathrm{E} 9(-5)$ \\
0.74 & $7.48780(-5)$ & $6.28357(-5)$ & $2.11675(-5)$ \\
0.78 & $1.05601(-4)$ & $8.95217(-5)$ & $2.83166(-5)$ \\
0.84 & $1.42325(-4)$ & $1.26916(-4)$ & $3.86510(-5)$ \\
0.90 & $1.78405(-4)$ & $1.74664(-4)$ & $5.25178(-5)$ \\
0.96 & $2.04635(-4)$ & $2.32369(-4)$ & $6.93219(-5)$ \\
1.07 & $2.03555(-4)$ & $3.40121(-4)$ & $1.05676(-4)$ \\
1.16 & $1.57548(-4)$ & $4.26999(-4)$ & $1.42452(-4)$ \\
1.26 & $8.17849(-5)$ & $5.00713(-4)$ & $1.87434(-4)$ \\
1.36 & $1.48070(-5)$ & $5.53810(-4)$ & $2.40734(-4)$ \\
1.47 & $7.07154(-6)$ & $5.73287(-4)$ & $2.99355(-4)$ \\
1.50 & $2.06596(-5)$ & $5.74057(-4)$ & $3.15400(-4)$ \\
1.66 & $2.10974(-4)$ & $5.39830(-4)$ & $4.02443(-4)$
\end{tabular}

Table 1 shows the results of the partial elastic cross sections of the positron collision with helium ion (in $\pi a_{0}^{2}$ ). The results show that the $\mathrm{P}$ - and $\mathrm{D}$-waves have the dominant contributions to the total elastic cross sections at $\mathrm{k}>1.0 a_{o}^{-1}$, while the $\mathrm{S}$-wave play the dominant role below $\mathrm{k}=1.0 \mathrm{a}_{o}{ }^{-1}$. Also, the results show the oscillating behavior of the $\mathrm{S}$-wave elastic cross sections, which supports the possible appearance of resonance at low energy below the positronium formation threshold especially at $\mathrm{k}>0.6 \mathrm{a}_{o}^{-1}$.
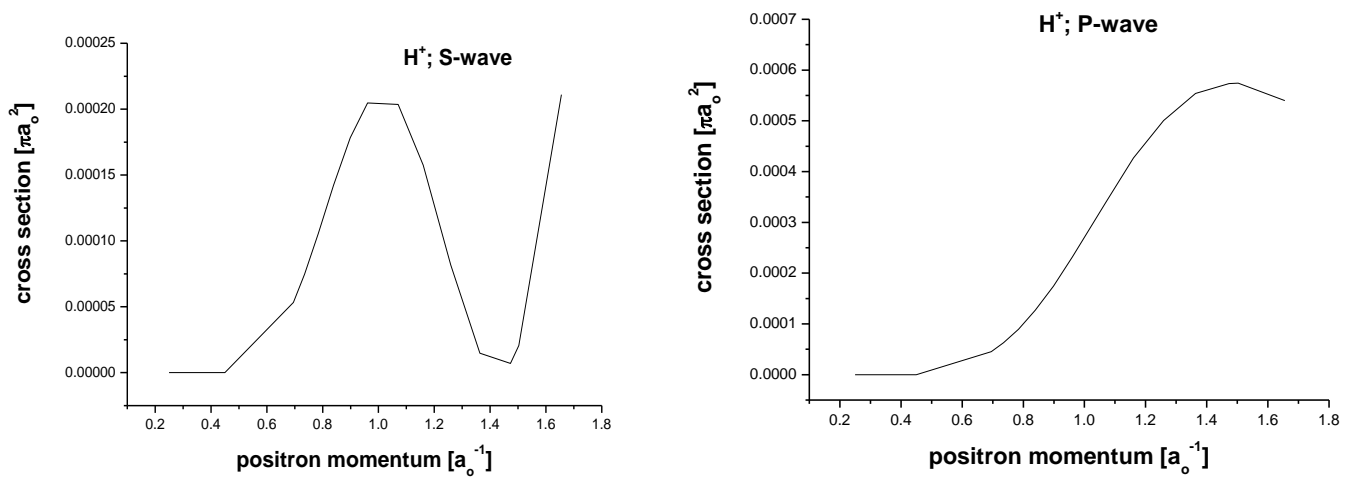

Figure 1. Partial elastic cross sections ( in $\pi a_{0}^{2}$ ) of positron- $\mathrm{He}^{+}(1 \mathrm{~s})$ scattering as a function of incident positron momentum for $\mathrm{S}$ - and $\mathrm{P}$-waves.

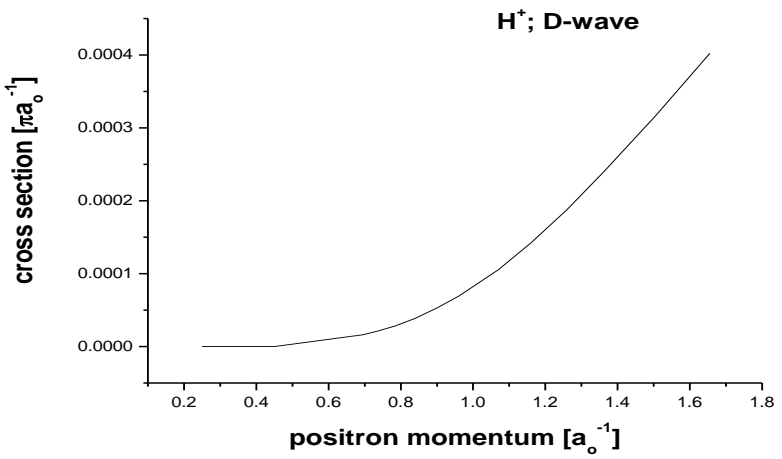

Figure 2. Partial elastic cross sections ( in $\pi a_{0}^{2}$ ) of positron- $\mathrm{He}^{+}(1 \mathrm{~s})$ scattering as a function of incident positron momentum for D-waves. 


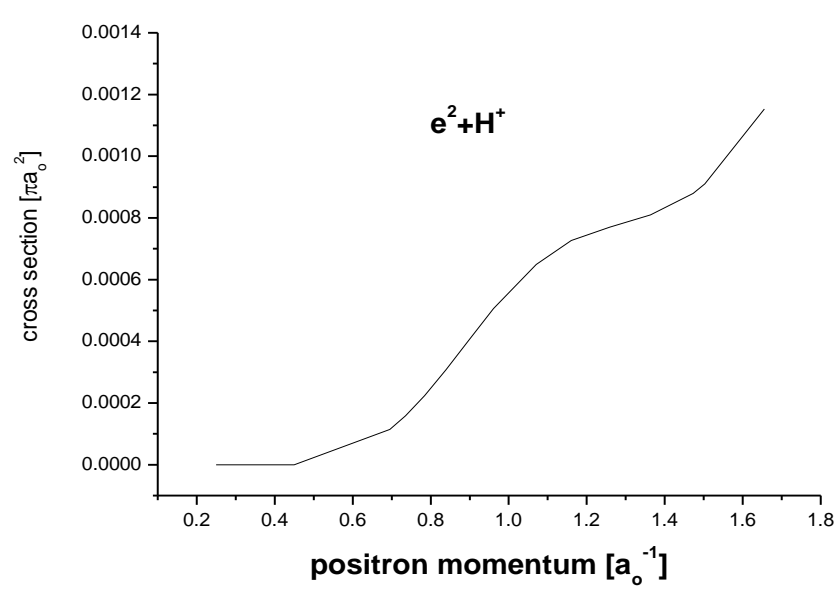

Figure 3. Total cross sections (in $\pi a_{0}^{2}$ ) of positron- $\mathrm{He}^{+}(1 \mathrm{~s})$ scattering as a function of incident positron momentum.

Fig. 1 and Fig. 2 show the role played by each partial wave in the total elastic cross sections. These figures also demonstrate the oscillating behavior of the cross sections in S-wave, where the existence of a resonance is expected, the first peak starts in S-wave and moving toward higher values of $k$ as the angular momentum L increases. Fig. 3 shows smoothly increasing in the cross section as the positron momentum increases. The main contribution of total cross section as positron momentum $k$ is greater than $1.0 a_{\mathrm{o}}^{-1}$ comes from P-wave and D-wave cross sections.

\section{CONCLUSION}

In this paper, we studied the elastic collisions of positrons with helium ion $\mathrm{He}^{+}(1 \mathrm{~s})$ in ground state. The incident positron energy is considered up to $40 \mathrm{eV}$ (positron momentum $k \cong 1.7$ a.u.). We used Harris-Nesbet variational method to determine the transition matrix that is used to calculate the scattering cross-section. We calculated the total cross sections that correspond to the $S$-, $P$-, and $D$-wave scatterings. The results show that the $P$ - and $D$-waves have large contributions to the total elastic cross sections at $k>1.0 a_{o}^{-1}$, while the $S$-wave play a dominant role below $k=1.0 a_{o}^{-1}$. The oscillating behavior of the S-wave elastic cross sections, supports the possible appearance of resonance at low energy below the positronium formation threshold especially at $k>0.6$ $a_{o}{ }^{-1}$. This result encourages the experimental physicists to carry out further positron-ion experiments.

\section{REFERENCES}

[1] D. G. Green, and G. F. Gribakin Phys. Rev. A 88, 032708 (2013)

[2] Y K Ho and Z-C Yan. Phys. B: At. Mol. Opt. Phys. 42044006 (2009).

[3] P.S. Mazumdar and A.S. Ghosh, Phys. Rev. A34, 4433 (1986).

[4] S. A. Novikov, M. W. J. Bromley, and J. Mitroy. Phys.Rev A 69, 052702 (2004).

[5] B H Bransden, C J Noble, and R J Whitehead, J. Phys. B: At. Mol. Opt. Phys. 342267 (2001).

[6] M. A. Abdel-Raouf, Journal of Physics B21, 2331 (1988).

[7] A.V. Lugovskoy, A.S. Kadyrov, I. Bray and A.T. Stelbovics, Phys.Rev.A82, 062708 (2010).

[8] F. E. Harris, Phys. Rev. Lett. 19: 173, 1967

[9] R K Nesbet, "Variational Method in Electron-Atom Scattering Theory" (New York: Plenum) (1980).

[10] T T Gien, 1995 Phys. Rev. A 521186.

[11] T.S. Stein, M.Harte, J.Jiang, W.E.Kauppil, C.K.Kwan, H. Li and S. Zhou, Nucl. Inst. Meth. B143, 68 (1998). 